\title{
Relation Between Daily Self-Weighing and Physique, Lifestyle Factors, and Glycemic Parameters in Japanese College Students
}

\author{
Tetsuya Kakuma ${ }^{\mathrm{a}, \mathrm{d}}$, Seiko Ariki ${ }^{\mathrm{b}}$, Yuichi Yoshida ${ }^{\mathrm{b}}$, Hirotaka Shibata ${ }^{\mathrm{b}}$, \\ Takashi Tsutsumic ${ }^{\mathrm{c}}$, Yoshikuni Kudo ${ }^{\mathrm{c}}$
}

\begin{abstract}
Background: The impact of self-weighing on young adults attending college has not been fully clarified. The present study aimed to examine self-weighing behavior and associated gender differences in Japanese college students and investigate the relation between habitual daily self-weighing and physique, lifestyle factors and glycemic parameters.
\end{abstract}

Methods: We retrospectively evaluated data from a 75-g oral glucose tolerance test (OGTT) that was performed during clinical training of medical students between 2010 and 2014. Information concerning physique (e.g. body weight and waist circumference) and a lifestylefocused questionnaire were completed before the OGTT. Participants comprised 441 fifth-grade university students ( 279 males and 162 females) from the Oita University Faculty of Medicine.

Results: A total of $26.8 \%$ of participants were habitual self-weighers. Females were significantly more likely to engage in habitual selfweighing than males. There were no significant differences in glucose metabolism between individuals who regularly weighed themselves and those who did not in either gender. Factors related to the performance of daily self-weighing were not skipping breakfast in males and regular exercise in females.

Conclusions: Although there was no meaningful relation between habitual self-weighing and glucose metabolism in this study, eating breakfast regularly and exercising regularly independently promoted self-weighing behavior in males and females, respectively. These findings suggest that these common practices may be helpful for col-

Manuscript submitted December 18, 2019, accepted December 23, 2019

aHealth Support Center, Hasama Campus, Oita University, Yufu City, Oita 879-5593, Japan

bDepartment of Endocrinology, Metabolism, Rheumatology and Nephrology, Faculty of Medicine, Oita University, Yufu City, Oita 879-5593, Japan

'Health Support Center, Dannoharu Campus, Oita University, Oita City, Oita 870-1192, Japan

${ }^{\mathrm{d}}$ Corresponding Author: Tetsuya Kakuma, Health Support Center, Hasama Campus, Oita University, 1-1 Idaigaoka, Hasama-machi, Yufu City, Oita 8795593, Japan.Email: kakuma@oita-u.ac.jp

doi: https://doi.org/10.14740/jem633 lege students as a method to encourage habitual daily self-weighing, consequently providing a healthy environment in college life to prevent potential obesity-related diseases.

Keywords: Self-weighing; College student; Glucose metabolism; Gender difference; Not skipping breakfast; Regular exercise

\section{Introduction}

Postadolescent university students are young adults in a developmental period associated with various lifestyle changes. The transition from puberty to adulthood often triggers unhealthy behaviors such as binge drinking, skipping breakfast and poor sleep habits. These changes are known to contribute to weight gain [1]. Weight control during this transitional period has an important implication for the pathogenesis of lifestyle-related diseases associated with obesity [2]. Most reports regarding self-weighing indicate that this behavior is a positive strategy for the treatment and prevention of obesity in adults [3, 4]. Frequent self-weighing has been associated with weight gain prevention as well as weight loss and weight loss maintenance. This behavior has also been associated with greater weight loss in younger generations with obesity [5] and healthy weight management in participants with normal weight [6]. The metabolic parameters of individuals who practice regular selfweighing may be better than those of individuals who do not practice self-weighing. Our previous study showed that daily self-weighing contributes to favorable glycemic control independent of prospective weight loss [7]. This finding suggests that a habit of self-weighing per se might be associated with improved glucose metabolism even though self-monitoring of body weight was not used as an intervention strategy. However, few studies have examined the relation between glucose metabolism and self-weighing as a habitual practice as opposed to a therapeutic strategy.

Moreover, the actual situation of self-weighing in young adults attending college has not been fully clarified, especially in Japan. A white paper was written about the current health state of university students based on various information obtained from regular health examinations in Japanese national universities in 2015 [8]. Recent data from approximately 
440,000 university students showed the percentage with certain daily habits (e.g. smoking, drinking and regular exercise) and reported a relationship between eating breakfast and smoking rates, drinking rates, habitual exercise, and sleeping time as well as an association between such lifestyle factors and physical examination parameters (e.g. body mass index (BMI), blood pressure and heart rate) [8]. However, to the best of our knowledge, no previous studies in young adults have examined the relation between habitual self-weighing and physique, lifestyle factors and glycemic parameters obtained from an oral glucose tolerance test (OGTT).

The present study aimed to provide more information on self-weighing in young adults attending college by examining 1) the current status of self-weighing behavior and related gender differences and 2) the relation between daily self-weighing and physique, lifestyle factors, and glycemic parameters obtained from a 75-g OGTT. Findings from this study may help with the introduction of a unique approach for medical staff at health support centers in universities to help students avoid future obesity-related lifestyle diseases.

\section{Materials and Methods}

\section{Subjects}

We retrospectively evaluated data from a 75-g OGTT performed during clinical training for medical students at Oita University Faculty of Medicine between 2010 and 2014. Selfreported information concerning physique (e.g. body weight and waist circumference) and a lifestyle-focused questionnaire were collected before administration of an OGTT. Participants comprised 441 fifth-grade university students (279 males and 162 females). Informed consent for the OGTT and for collecting information concerning physique and lifestyle behaviors was obtained from all students while in school. The present study was conducted in accordance with the Declaration of Helsinki and was carried out with the approval of the Ethics Committee of Oita University (date of approval: January 16, 2017, approval number: 1146).

\section{Assessment of variables}

A questionnaire regarding lifestyle behaviors was administered to all participating students the day before the OGTT. Current smoking was classified as yes or no (including nonsmokers and past smokers). Regular exercise was classified as yes (member of an athletic club or exercising more than 3 days during a week) or no. Alcohol drinking was classified as yes (including occasional drinkers and drinking more than 1 day a week) or no (nondrinker). Skipping breakfast was classified as yes (no breakfast more than 3 days a week) or no. Frequently eating late at night was classified as yes (night eating after $10 \mathrm{pm}$ more than 3 days a week) or no. Excessive soft drink consumption was classified as yes (more than $500 \mathrm{~mL}$ of soft drink intake more than 3 days a week) or no. Daily selfweighing was classified as yes (including morning once a day, evening once a day and more than once a day) or no (none). The rare cases of self-weighing less than once a day, such as weekly or monthly, were combined into the "none" category. Gender, age, height and weight were self-reported. Waist circumference at the umbilical level was measured by a fellow medical student in the late exhalation phase according to the recommendation proposed by the Japanese Committee for the diagnostic criteria of metabolic syndrome [9].

After confirming that the participating students did not eat and drink after $11 \mathrm{pm}$ on the previous day, and that they had skipped breakfast on the day of the OGTT, blood samples were collected by a fellow medical student at 0, 30, 60 and 120 min, according to a standard 75-g OGTT protocol [10]. Participants were given instructions on OGTT training at a meeting the day before the test. Clinical practice leaders taught the students how to draw blood from a vein. This practical training was completed on the morning of the OGTT.

\section{Laboratory measurements}

Plasma glucose and insulin levels were measured using standard laboratory procedures at the clinical laboratory at Oita University Hospital. Insulin resistance and secretion were determined by homeostasis model assessment [11] based on fasting plasma glucose and insulin values: homeostasis model assessment of insulin resistance $(\mathrm{HOMA}-\mathrm{IR})=$ fasting glucose $(\mathrm{mg} / \mathrm{dL}) \times$ fasting insulin $(\mu \mathrm{U} / \mathrm{mL}) / 405$; homeostasis model assessment of beta cell function $(\mathrm{HOMA}-\beta)=360 \times$ fasting insulin $(\mu \mathrm{U} / \mathrm{mL}) /$ fasting glucose $(\mathrm{mg} / \mathrm{dL})-63$. The insulinogenic index was calculated as the following incremental ratio: $\Delta$ insulin $/ \Delta$ glucose $=$ insulin at $30 \mathrm{~min}(\mu \mathrm{U} / \mathrm{mL})$ - fasting insulin $(\mu \mathrm{U} / \mathrm{mL}) /$ glucose at $30 \mathrm{~min}(\mathrm{mg} / \mathrm{dL})$ - fasting glucose $(\mathrm{mg} /$ $\mathrm{dL}$ ). The area under the curve (AUC) for glucose and insulin during the OGTT was calculated with the trapezoidal rule. The glucose tolerance category was determined according to the Japanese Diabetes Association criteria for the 75-g OGTT [12]: diabetes was defined as fasting glucose $\geq 126 \mathrm{mg} / \mathrm{dL}$ and/ or 2-h glucose $\geq 200 \mathrm{mg} / \mathrm{dL}$, impaired glucose tolerance (IGT) was defined as fasting glucose $\geq 110 \mathrm{mg} / \mathrm{dL}$ but $<126 \mathrm{mg} / \mathrm{dL}$ and/or 2-h glucose $\geq 140 \mathrm{mg} / \mathrm{dL}$ but $<200 \mathrm{mg} / \mathrm{dL}$, and normal glucose tolerance was defined as fasting glucose $<110 \mathrm{mg} / \mathrm{dL}$ and 2-h glucose $<140 \mathrm{mg} / \mathrm{dL}$. BMI was calculated as weight $(\mathrm{kg})$ divided by height squared $\left(\mathrm{m}^{2}\right)$. Obesity was characterized by a BMI $\geq 25 \mathrm{~kg} / \mathrm{m}^{2}$, and abdominal obesity was defined as a waist circumference $\geq 85 \mathrm{~cm}$ in males and $\geq 90 \mathrm{~cm}$ in females [9].

\section{Statistical analysis}

Because all values were not normally distributed, data are expressed as medians (25th - 75th percentiles) and were nonparametrically compared between groups using the Wilcoxon rank-sum test. Differences in proportions were compared using the $\chi^{2}$ test or Fisher's exact probability test. Univariate logistic regression analysis was used to investigate the relations between habitual daily self-weighing and clinical characteristics. 
Table 1. Clinical Characteristics and Lifestyle Profiles of Study Participants

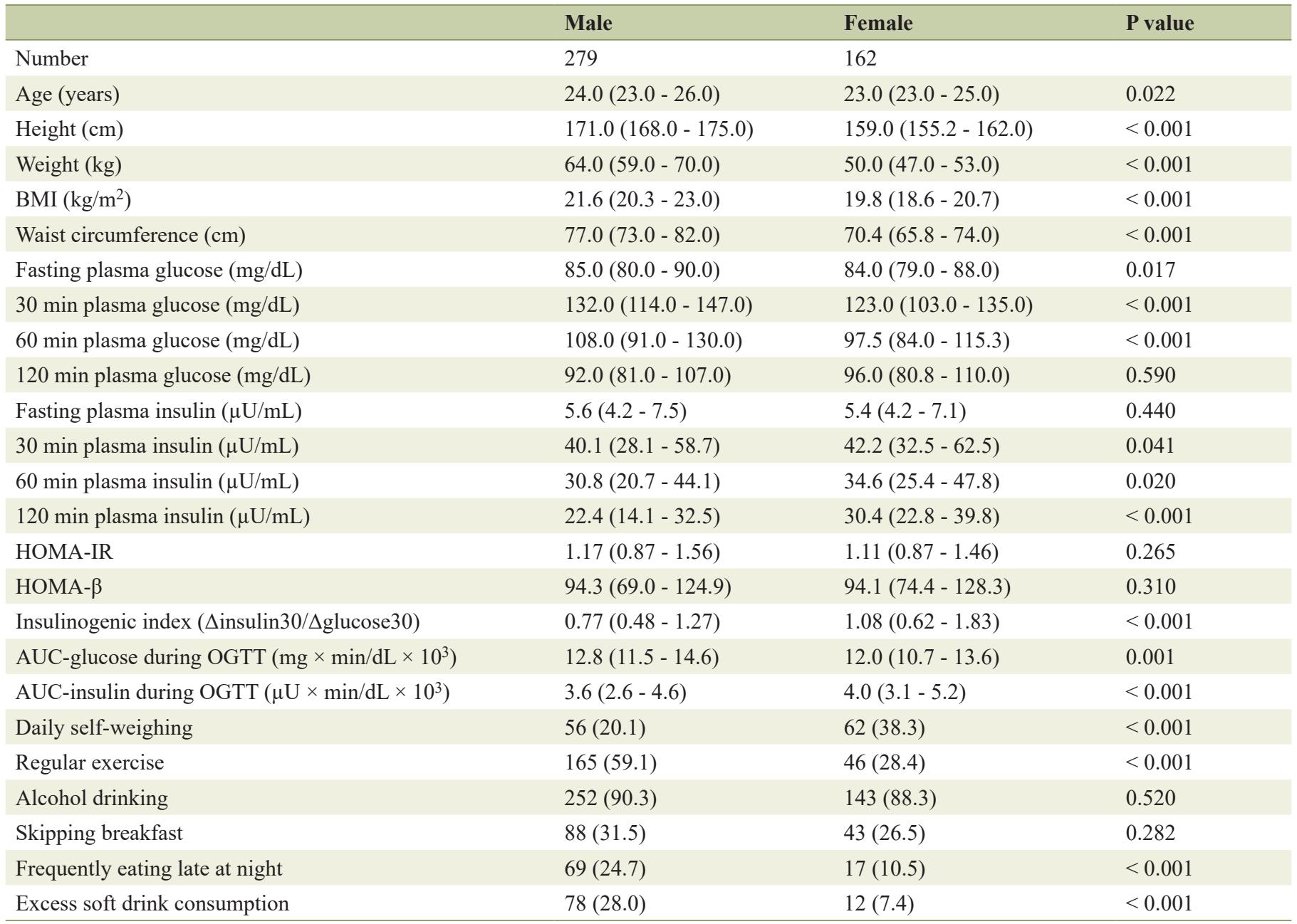

Figures in parentheses are interquartile ranges (25th - 75th percentiles) or $n$ (\%). BMI: body mass index; HOMA-IR: homeostasis model assessment of insulin resistance; HOMA- $\beta$ : homeostasis model assessment of beta-cell function; AUC: area under the curve; OGTT: oral glucose tolerance test.

Lifestyle statuses were represented by dummy variables $(1=$ yes, $0=$ no). Next, multivariate logistic regression analysis was conducted to examine factors possibly affecting habitual daily self-weighing. To build this model, factors with $\mathrm{P}<0.1$ in the univariate logistic regression analysis were included in the multivariate analysis. For clinical usability of the gender comparison, weight (as opposed to BMI and/or waist circumference), was selected as the explanatory variable for physique. Statistical analyses were performed using JMP software programs (version 12.0.1 for Macintosh, SAS Institute Inc., Cary, NC, USA). A P value $<0.05$ (two-sided) was considered significant.

\section{Results}

\section{Clinical characteristics and lifestyle profiles}

Clinical characteristics and lifestyle profiles of the participants are shown in Table 1 . Males were significantly older than females $(\mathrm{P}<0.05)$ and had a significantly greater height, weight, $\mathrm{BMI}$ and waist circumference $(\mathrm{P}<0.001, \mathrm{P}<0.001, \mathrm{P}<0.001$ and $\mathrm{P}<0.001$, respectively). A total of $18.6 \%$ of males were diagnosed with abdominal obesity; however, none of the females met that classification (data not shown). The fasting plasma glucose, 30-min postload glucose, 60-min postload glucose and AUC-glucose during the OGTT were significantly greater in males $(\mathrm{P}<0.05, \mathrm{P}<0.001, \mathrm{P}<0.001$ and $\mathrm{P}$ $<0.005$, respectively), and the 30 -min postload insulin, 60min postload insulin, 120-min postload insulin, insulinogenic index and AUC-insulin during the OGTT were significantly greater in females $(\mathrm{P}<0.05, \mathrm{P}<0.05, \mathrm{P}<0.001, \mathrm{P}<0.001$ and $\mathrm{P}<0.001$, respectively). There were no significant differences in HOMA-IR or - $\beta$ between males and females. IGT was identified in $4.7 \%$ of males and $3.7 \%$ of females; however, there was no significant difference in the prevalence of IGT between the genders. A total of $26.8 \%$ of participants reported weighing themselves at least once a day. These individuals were defined as having a habit of daily self-weighing. Self-weighing fre- 
quency was significantly higher in females than in males $(\mathrm{P}<$ 0.001). The proportions of current smoking (data not shown), regular exercise, frequently eating late at night and excessive soft drink consumption were significantly greater in males than in females ( $\mathrm{P}<0.001, \mathrm{P}<0.001$ and $\mathrm{P}<0.001$, respectively). Only one female in the study was a current smoker. Because she could be easily identified, data regarding current smoking were omitted from the tables on ethical grounds.

\section{Clinical characteristics and lifestyle factors according to daily self-weighing}

When students were divided according to whether or not they performed daily self-weighing (Table 2), the weight, BMI and waist circumference of participants were significantly higher in the self-weighing group in males $(\mathrm{P}<0.005, \mathrm{P}<0.001$ and $\mathrm{P}$ $<0.005$, respectively), whereas in females, a trend was shown only for weight and BMI. There were no significant differences between the groups according to self-weighing status ( $\mathrm{P}$ $=0.062$ and $\mathrm{P}=0.064$, respectively). In males, the percentage of participants who skipped breakfast was significantly lower in the self-weighing group $(\mathrm{P}<0.05)$, whereas in females, the self-weighing group was significantly younger and participated in significantly more physical activity than the non-selfweighing group ( $\mathrm{P}<0.05$ and $\mathrm{P}<0.05$, respectively). There were no significant differences in glucose metabolism between the groups in either gender.

\section{Relation between clinical characteristics and lifestyle with daily self-weighing}

Because the clinical characteristics and lifestyle profiles were significantly different between the male and female students, the relation of age, physique and lifestyle with daily selfweighing was examined by gender using logistic regression analysis. Regarding the variables for physique, we used weight because it is not necessary to calculate, and the changes per unit are easy to assess. In males, daily self-weighing was significantly associated with age, weight and skipping breakfast ( $\mathrm{P}<0.05, \mathrm{P}<0.01$ and $\mathrm{P}<0.05$, respectively) (Table 3$)$. The odds ratio for skipping breakfast was the lowest among those factors. Multivariate logistic regression analysis was conducted to investigate the association between daily self-weighing and skipping breakfast after adjusting for potential confounders, including age and frequently eating late at night. Skipping breakfast was independently and negatively related to daily self-weighing in males $(\mathrm{P}<0.05)$. In addition, weight was also independently and significantly associated with daily selfweighing in males $(\mathrm{P}<0.05)$.

In females, daily self-weighing was significantly associated with current weight and regular exercise $(\mathrm{P}<0.05$ and $\mathrm{P}<$ 0.01 , respectively) (Table 4 ). The odds ratio of regular exercise was two-fold higher than that of current weight. To investigate the association between daily self-weighing and regular exercise, multivariate logistic regression analysis was also performed after adjusting for potential confounders, including age and skipping breakfast. Regular exercise was independently and positively related to daily self-weighing in females $(\mathrm{P}<$ $0.05)$.

\section{Discussion}

The main findings of the present study are as follows. 1) A total of $26.8 \%$ of college students enrolled in the present study were habitual self-weighers who weighed themselves at least once daily. Females were more likely to report self-weighing behaviors than males. 2) In young adults attending college, there were no significant differences in glucose metabolism between groups with and without self-weighing habits. The current weight of the participants was significantly higher in the self-weighing group in males, while in females, results showed only a trend for this variable. Factors related to daily self-weighing were not skipping breakfast and weight gain in males and exercise habits in females.

There are few reports about the importance of "daily" self-weighing for health management, especially in college students. In Western countries, the proportion of daily selfweighing was reported to be less than $5 \%$ in young adults $(\mathrm{n}=$ 2,287 , mean age $=25.3$, female $=55.0 \%$, overweight or obese $=51.5 \%$ ) [13] and approximately $11 \%$ in working adults $(\mathrm{n}=$ 2,732 ) [14]. Daily self-weighers comprised $26.8 \%$ of the students in this study $(n=441$, mean age $=24.8$, female $=36.7 \%$, mean BMI $=21.2$ ), and the percentage was almost the same as that $(25.2 \%)$ in our previous report $(n=306$, mean age $=62.3$, female $=37.3 \%$, mean BMI $=24.6$ ) that assessed daily selfweighing in patients with type 2 diabetes [7]. The Health Support Center at Tokai University also reported that daily selfweighers comprised almost $23 \%$ of the participants enrolled in a health investigation [15]. In general, it appears that Japanese people are likely to weigh themselves more frequently than Western people. This may represent a fundamental difference in lifestyle behavior for health management between Japan and Western countries.

The prevalence of daily self-weighing was higher in females $(31 \%)$ than in males $(15 \%)$ in Tokai University's report related to working adults described above [15]. In college students, the ratio of daily self-weighing between females and males in our study was almost the same (38.3\% vs. $20.1 \%$, respectively) as that noted in working adults and was similar to the estimates of daily self-weighing among Western young adults $(6.3 \%$ of females and $2.7 \%$ of males) [13]. The high rate in females may be due to a high level of interest in body image; however, there were no significant differences in body weight between groups with and without self-weighing habits in this study. In males, body weight and waist circumference were significantly higher in the group with self-weighing habits, and current weight per se was a significant independent promoter of daily self-weighing in males. These findings imply males are preoccupied with their bodies. However, it is possible that the high rate of self-weighing in females had already prevented weight gain in this group. Although the reasons the prevalence of daily self-weighing was higher in females than in males were not clarified in the present study, it may be interesting to 


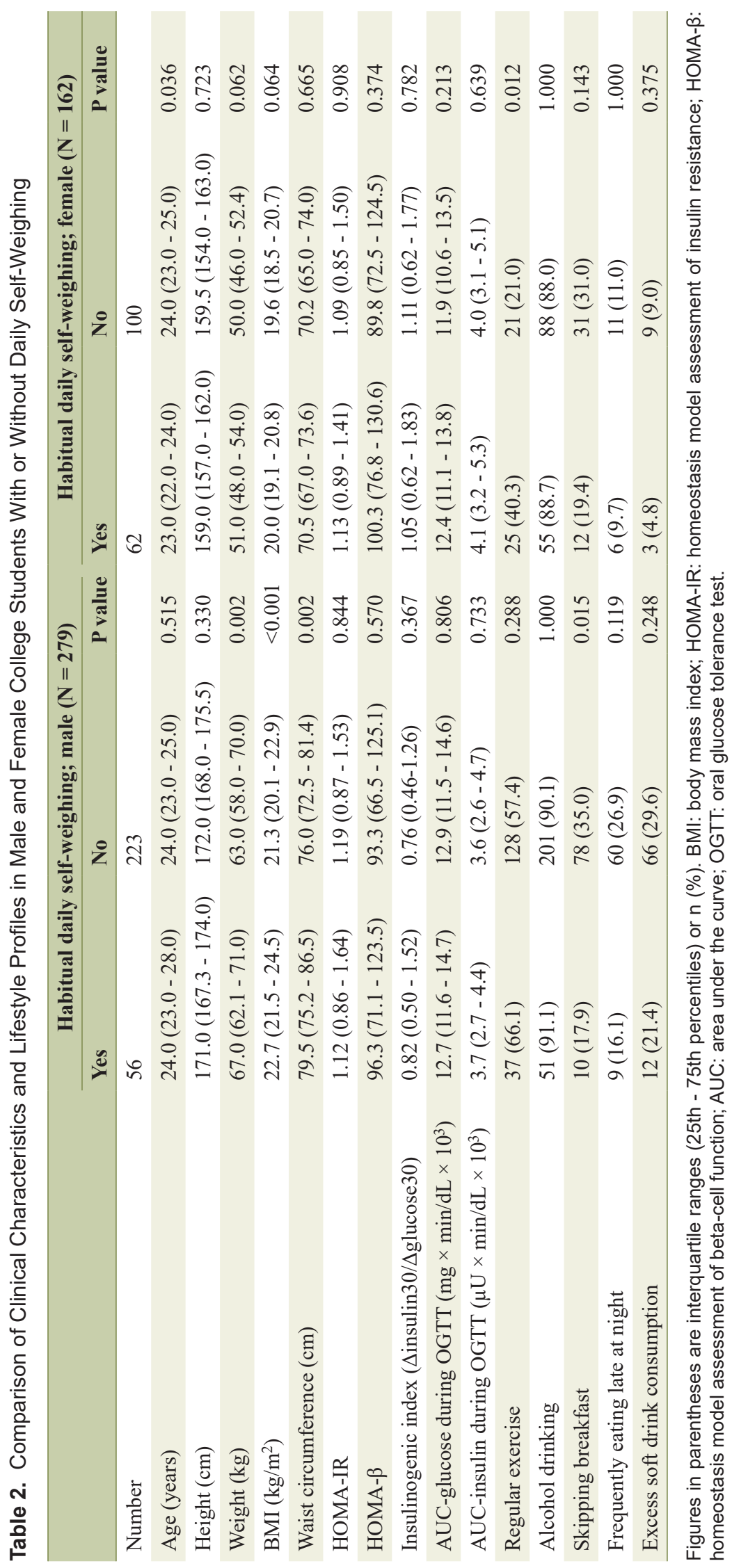




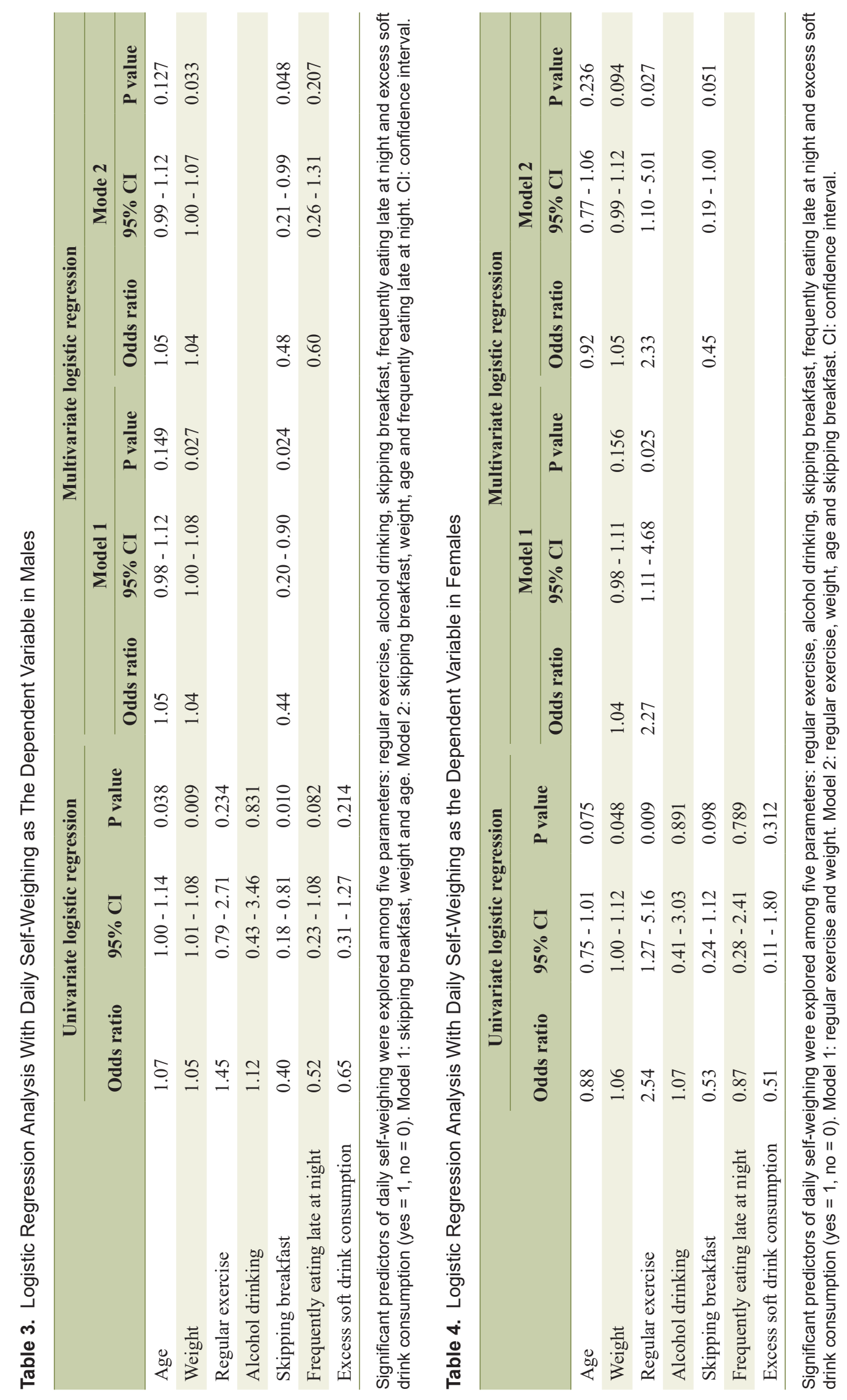


evaluate whether minimal weight changes lead to an increase or decrease in self-weighing habits in college students who are mostly normal-weight individuals.

Our previous study demonstrated that daily self-weighing contributes to favorable glycemic control independent of prospective weight loss [7]. However, the relationship between habitual self-weighing and glucose metabolism was not observed in the present study. There are some possible reasons for the difference in results between the studies of working adults and college students. First, the students in the present study were not as old as subjects enrolled in previous studies. There is an essential difference in glucose tolerance between late middle-aged subjects and young postadolescent subjects. This may be one of the reasons that glucose metabolism of students with daily self-weighing habits was not different from that of students without daily self-weighing habits. The other explanation is associated with the difference between patients with type 2 diabetes and generally healthy students. Therefore, the benefit of self-weighing might not be observed in the students whose IGT was only $4.3 \%$.

A recent longitudinal observational study of persons with a mean age of 24 years showed that only a quarter of young adults maintained their body weight over 10 years, $68.3 \%$ of participants gained more than $5 \%$ of their weight and eating regularly and having no history of excessive dieting (intentionally losing $\geq 5 \mathrm{~kg}$ ) were significant factors related to successful weight maintenance in both genders [16]. This means that continuous regular eating habits are even more essential to successful weight management. In the present study, skipping breakfast was independently and significantly related to daily self-weighing habits in males. The logistic regression analysis suggests that not skipping breakfast may be a potent factor for making daily self-weighing a lifelong habit in males. If breakfast is not eaten, blood glucose levels will rise after lunch, which is referred to as the second-meal phenomenon [17]. Thus, regularly eating breakfast is important for maintaining favorable glucose metabolism from a standpoint of chrononutrition. Because of the cross-sectional study design, it is impossible to determine a cause-and-effect relationship between self-weighing and not skipping breakfast. However, it is possible that regularly eating breakfast synergistically activated a healthy lifestyle by promoting daily self-weighing. The ratio of college students who exercise regularly, eat late at night and consume soft drinks is generally higher in males than in females [8]. These tendencies were confirmed in our study. Primarily, the reason the ratio of college students who eat late dinners and consume excessive soft drinks was higher in males may be because a higher number of males participated in athletic club activities or regularly exercised. In terms of healthy behaviors, exercise was more common in males, whereas good eating habits and not smoking were more common in females. It is interesting that regular exercise was independently and significantly related to daily self-weighing in females. In the present study, females more commonly tended not to eat late at night or drink excessive amounts of soft drink, which suggests they placed more importance on eating behaviors rather than exercise. Therefore, regular exercise may increase the motivation for self-weighing in females. As mentioned above, habitual self-weighing accompanied by regularly eating breakfast and exercising would synergistically be expected to prevent the pathogenesis of lifestyle-related diseases related to obesity, including type 2 diabetes, in college students.

There are several limitations to this study. Height and weight were self-reported to avoid measuring body weight in front of everyone, which was especially important for the female students. A recent study of 50 female students (mean age $=21$ years) in Japan demonstrated that self-reported height and weight were reliable because intraclass correlation coefficients between self-reported height and weight and those measured with scales were above 0.9 [18]. However, to check the accuracy of our study, we compared self-reported height and weight to those measured with scales in routine physical exams at our university. Although the participants, especially females, may underreport their measurements, there were no statistically significant differences between the self-reported data and health examination data (males vs. females: 171.3 (167.6 - 175.4) vs. 159.4 (155.3 - 162.8) in height; $\mathrm{P}<0.001 ; 64.9$ (59.7 - 70.8) vs. 51.5 (47.9 - 55.1) in weight; $\mathrm{P}<0.001)$, indicating that the self-reported data did not influence the results of this study. We cannot deny the possibility that the weights of participants changed from the time of the routine exam in spring. In any case, further observational studies with a larger sample size are necessary to confirm these results. Some studies showed that frequent self-weighing caused an increase in anxiety and depression in younger females and predicted binge eating and unhealthy weight control behavior [13, 19]. Because questionnaires regarding unhealthy behaviors such as binge eating and family history of diabetes were not administered to students in the present study, further studies should be conducted to examine ways to refine such types of interventions to improve their efficacy.

In conclusion, a habit of regular self-weighing may be an indicator of healthy individuals. Male students who skipped breakfast and the female students who had a sedentary lifestyle were less likely to perform habitual self-weighing. Self-weighing is a simple technique that can be used not only for weight loss in individuals who are overweight or obese but also for weight maintenance in normal-weight populations. Weight monitoring provides an opportunity for positive feedback for students to identify how specific situations or patterns of eating or physical activity relate to changes in body weight. Eating breakfast for males and exercising regularly for females, along with habitual self-weighing, may synergistically help establish a healthy lifestyle.

\section{Acknowledgments}

We thank Keishi Mori for his assistance with the statistical analysis.

\section{Financial Disclosure}

This research did not receive any specific grant from funding agencies in the public, commercial, or not-for-profit sectors. 


\section{Conflict of Interest}

The authors declare that there are no conflict of interest.

\section{Informed Consent}

Informed consent for the OGTT and for collecting information concerning physique and lifestyle behaviors was obtained from all students while in school. Because students have already graduated, we announced that the opportunity to opt out was always available by providing the information on the website of our department. To date, there have been no inquiries.

\section{Author Contributions}

Tetsuya Kskuma contributed to conceptualization, methodology, software, formal analysis, investigation, writing original draft and visualization. Seiko Ariki and Yuichi Yoshida contributed to investigation and data curation. Hirotaka Shibata contributed to supervision and project administration. Takashi Tsutsumi and Yoshikuni Kudo contributed to writing review and editing. All authors read and approved the final manuscript.

\section{Data Availability}

The data supporting the findings of this study are available from the corresponding author upon reasonable request.

\section{References}

1. Nelson MC, Story M, Larson NI, Neumark-Sztainer D, Lytle LA. Emerging adulthood and college-aged youth: an overlooked age for weight-related behavior change. Obesity (Silver Spring). 2008;16(10):2205-2211.

2. Examination Committee of Criteria for 'Obesity Disease' in Japan, Japan Society for the Study of Obesity. New criteria for 'obesity disease' in Japan. Circ J. 2002;66(11):987-992.

3. Linde JA, Jeffery RW, French SA, Pronk NP, Boyle RG. Self-weighing in weight gain prevention and weight loss trials. Ann Behav Med. 2005;30(3):210-216.

4. Pacanowski CR, Levitsky DA. Frequent self-weighing and visual feedback for weight loss in overweight adults. J Obes. 2015;2015:763680.

5. Gokee-Larose J, Gorin AA, Wing RR. Behavioral selfregulation for weight loss in young adults: a randomized controlled trial. Int J Behav Nutr Phys Act. 2009;6:10.
6. Rosenbaum DL, Espel HM, Butryn ML, Zhang F, Lowe MR. Daily self-weighing and weight gain prevention: a longitudinal study of college-aged women. J Behav Med. 2017;40(5):846-853.

7. Kakuma T, Chiba S, Takahashi Y, Kai K, Tsubone T, Yoshimatsu H. Self-weighing contributes to glycemic control: importance of measuring body weight. Diabetol Int. 2013;4:40-44.

8. Japanese National University Council of Health Administration Facilities. White paper: University Student Health 2015. Nagoya: Nagoya University Co-op; 2018. Japanese.

9. Anonymous. Metabolic Syndrome Definition and Diagnostic Criteria in Japan. J Jpn Soc Int Med. 2005;94:794809. Japanese.

10. Oka R, Yagi K, Sakurai M, Nakamura K, Moriuchi T, Miyamoto S, Nohara A, et al. Insulin secretion and insulin sensitivity on the oral glucose tolerance test (OGTT) in middle-aged Japanese. Endocr J. 2012;59(1):55-64.

11. Matthews DR, Hosker JP, Rudenski AS, Naylor BA, Treacher DF, Turner RC. Homeostasis model assessment: insulin resistance and beta-cell function from fasting plasma glucose and insulin concentrations in man. Diabetologia. 1985;28(7):412-419.

12. Japan Diabetes Society. Treatment Guide for Diabetes 2018-2019. Tokyo: Bunkodo, 2018. Japanese.

13. Quick V, Larson N, Eisenberg ME, Hannan PJ, NeumarkSztainer D. Self-weighing behaviors in young adults: tipping the scale toward unhealthy eating behaviors? J Adolesc Health. 2012;51(5):468-474.

14. Gavin KL, Linde JA, Pacanowski CR, French SA, Jeffery RW, Ho YY. Weighing frequency among working adults: cross-sectional analysis of two community samples. Prev Med Rep. 2015;2:44-46.

15. Tanabe N, Somemiya E, Iwamoto T, Ohta H. Daily selfweighers have a favorable laboratory data. Jpn J Health Educ Promot. 2010;18:92. Japanese.

16. Karkkainen U, Mustelin L, Raevuori A, Kaprio J, Keski-Rahkonen A. Successful weight maintainers among young adults-A ten-year prospective population study. Eat Behav. 2018;29:91-98.

17. Jovanovic A, Leverton E, Solanky B, Ravikumar B, Snaar $\mathrm{JE}$, Morris PG, Taylor R. The second-meal phenomenon is associated with enhanced muscle glycogen storage in humans. Clin Sci (Lond). 2009;117(3):119-127.

18. Satoh E, Araki K, Yamamoto Y. Reliability of reported height and weight for oneself. Jpn J Sports Rehabil. 2017;6:17-20. Japanese.

19. Pacanowski CR, Loth KA, Hannan PJ, Linde JA, Neumark-Sztainer DR. Self-weighing throughout adolescence and young adulthood: implications for well-being. J Nutr Educ Behav. 2015;47(6):506-515 e501. 